\title{
EL APRENDIZAJE SERVICIO, INTERCULTURALIDAD Y JUSTICIA SOCIAL: EXPERIENCIAS DISRUPTIVAS Y TRANSFORMADORAS CON FUTURAS MAESTRAS DE EDUCACIÓN INFANTIL Y PRIMARIA
}

\author{
Imanol Santamaría-Goicuria \\ Agurtzane Martinez Gorrochategi \\ Universidad de Mondragón-Mondragon Unibertsitatea
}

\section{Resumen}

El aprendizaje servicio se está mostrando como una metodología activa y socialmente transformadora de las prácticas tradicionales de enseńanza en la educación superior, con un gran valor para generar experiencias significativas de aprendizaje y promover el compromiso social de los futuros educadores. La experiencia de la que damos cuenta en este artículo se ha desarrollado durante el segundo cuatrimestre del curso 2016-2017 en la asignatura: "gestión de proyectos específicos", de $4 .^{\circ}$ curso del Grado de Magisterio Educación Infantil y Primaria. Ha participado un grupo de 54 alumnas en la Facultad de Educación y Deporte de la Universidad del País Vasco de Vitoria-Gasteiz. Como resultado de esta experiencia se evidencia que los futuros docentes demandan experimentar un modelo de formación verdaderamente comprometida con la sociedad y con ellos mismos para poder desarrollarse como personas justas, críticas, comprometidas con la comunidad, indistintamente del contexto de su localización, es decir, una educación desde y para la justicia social.

PALABRAS ClAVE: formación de profesorado, inclusión, metodologías activas, universidad, innovación educativa.

\section{SERVICE LEARNING, INTERCULTURALITY AND SOCIAL JUSTICE: \\ DISRUPTIVE AND TRANSFORMATIVE EXPERIENCES WITH FUTURE TEACHERS OF EARLY CHILDHOOD AND PRIMARY EDUCATION}

\section{Abstract}

Service learning is being shown as an active and socially transformative methodology of traditional teaching practices in higher education, with great value to generate meaningful learning experiences and promote the social commitment of future educators. The experience of which we give account in this article, was developed during the second term of the 20162017 course, in the subject: "Management of specific projects" of the 4th year of the Degree of Primary and Secondary Education. A group of 54 students participated in the Faculty of Education and Sport of the University of the Basque Country in Vitoria-Gasteiz. As a result of this experience, it is evident that future teachers demand to experience a training model that is truly committed to society and to themselves, in order to be able to develop as just and critical people committed to the community, regardless of the context of their location, from and for social justice.

KeYwords: Teacher training. Inclusion. Active methodologies. University. Educational innovation.

DOI: http://doi.org/10.25145/j.qurricul.2018.31.005

Revista Qurriculum, 31; junio 2018, pp. 97-118; ISSN: e-2530-8386 


\section{INTRODUCCIÓN}

Vivimos en un mundo repleto de incertidumbres descrito como modernidad líquida (Bauman, 2007). Un mundo que nos dificulta percibir lo cercano y lo lejano. Un mundo en donde el conocimiento que se genera, divulga, interpreta y se examina caduca rápidamente. Esta limitación conceptual sobre el futuro, lo repentino del aquí y ahora y las transformaciones en las últimas décadas en el ámbito educativo fruto del mundo Volátil, Incierto, Complejo y Ambiguo (VICA) (Sancho y Correa, 2013), invita a desarrollar una renovación de las metodologías utilizadas en la educación en general y más concretamente en la formación del profesorado.

Vemos necesario responder a las demandas actuales generadas por el no saber qué va a suceder mańana y la volatilidad del presente, que condicionan: (1) los procesos de enseñanza-aprendizaje, (2) lo que se espera de la educación en general y más concretamente de las universidades, (3) la composición de las nuevas sociedades supeditadas a los movimientos migratorios y (4) lo declarado por Alonso y Arandia (2013), Caride (2008); Rizvi (2010) y Rodicio (2010) sobre la necesidad de cambio del papel de la universidad y los docentes, problematizando la función de la misma. Demandamos una universidad con elementos educativos desde y para una formación global. Una universidad cuya oferta formativa contemple cualquier campo profesional y cualquier grupo de profesionales (Gehring, 2008 en Francisco y Moliner, 2010), que facilite el desarrollo de una acción de servicio a la comunidad y erradicar así las situaciones de injusticia y de desigualdad social fruto del modelo neoliberal en el que nos encontramos inmersos. En palabras de Francisco y Moliner (2010), un concepto de universidad como espacio y actor transformador de la sociedad.

Igualmente, aspiramos a que nuestros futuros maestros renueven la práctica docente. Queremos crear facilitadores de los procesos de enseñanza-aprendizaje de nuestros hijos. No deseamos crear meros transmisores de información, pues lo único que perpetúan son relaciones pedagógicas de poder justificadas por el hasta ahora conocimiento dominante y hegemónico. Ansiamos personas que acompañen y ayuden (cuando la situación lo requiera) en la coconstrucción personal de los educandos y que incidan en la importancia del sentir experimentado mediante el hacer, de las emociones, de las relaciones con el otro y de los valores que surgen de estas.

Por esta razón, creemos que la implementación de metodologías activas como la del Aprendizaje Servicio (ApS) posibilitan el desarrollo de sociedades más justas, pues facilitan la obtención de competencias no solo fundamentadas en la adquisición de conocimientos mediante la transmisión de los mismos (aprender a conocer), sino que de igual manera facilitan aquellas que Delors (1996) relaciona con lo afectivo y emocional (aprender a hacer, aprender a vivir y aprender a ser) y que son transcendentes en los procesos de construcción social y de desarrollo humano.

En este artículo, en consecuencia, desarrollamos los conceptos de Aprendizaje Servicio (ApS) y Justicia Social para analizar un proyecto llevado a cabo en la Facultad de Educación y Deporte de la Universidad del País Vasco y así poder establecer relaciones en el desarrollo de sociedades más justas mediante la imple- 
mentación de este tipo de metodologías, que, según Baldwin, Buchanan y Rudisill (2007) aumenta la conciencia de justicia social en los estudiantes.

\section{2. ¿POR QUÉ ESTA EXPERIENCIA?: DE LO MACRO A LO MICRO}

Existen diversos trabajos de investigación realizados sobre el ApS en otras universidades (Aramburuzabala, 2012; Lucas y Martínez-Odria, 2012) que enfatizan la notable repercusión que la implementación de esta metodología posee: (1) en la calidad del aprendizaje del alumnado, siendo este más profundo y significativo, (2) en las competencias profesionales y cívicas, (3) en las habilidades personales, sociales y cívicas; mejor autoestima y conocimiento de sí mismo, así como (4) en una mayor adquisición de responsabilidad social (Martínez, Martínez, Alonso y Gezuraga, 2013). Pero son pocas las experiencias narradas sobre la repercusión que esta metodología tiene en la formación del profesorado y muchas menos aquellas desarrolladas desde la perspectiva de justicia social (Martínez, 2008; Hernández, 2010; Aramburuzabala, 2012).

No podemos obviar la Responsabilidad Social Universitaria (RSU) en dicho proceso, que tal y como De Ferari (2006) define es la capacidad que tiene la universidad de difundir y poner en práctica un conjunto de principios y valores generales y específicos, por medio de diferentes procesos, en respuesta a las necesidades de la comunidad donde ésta se compromete a implementar estrategias capaces de mejorar el devenir de las comunidades donde se incluyan (Alonso-Sáez, Arandia, Martínez y Gezuraga, 2015).

De igual manera, la cantidad de alumnado migrante en las escuelas cada vez es mayor. Este incremento demanda un cambio relevante en la educación, donde, por poner un ejemplo, las personas migrantes no sean las únicas que únicamente se tengan que adecuar en los procesos de integración, sino que sea una responsabilidad compartida entre todos los integrantes de la sociedad (Coelho, 2006). Para ello, en la Comunidad Autónoma Vasca se ha desarrollado el Plan Estratégico de Atención a la Diversidad en el marco de una Escuela Inclusiva (2012-2015) y el Marco del Modelo Educativo Pedagógico, dentro del Plan Heziberri 2020, con la intención de conjugar las líneas de innovación y desarrollo establecidas en el marco europeo para el año 2020 con respecto a la educación y formación y los retos educativos propios de nuestro contexto y entorno (Correa y Santamaría-Goicuria, 2016).

Visto esto y teniendo en cuenta la temática general, «Interculturalidad y diversidad cultural», que trabajamos durante el último año de la titulación de Magisterio en la mención de Educación Intercultural, nos dimos cuenta de que el planteamiento de la titulación, aunque sí recaba la propuesta teórica establecida por el marco recogido en el Plan Heziberri 2020, no contaba con prácticas relevantes que facilitaran una adquisición significativa de tales competencias por parte del alumnado. Es por ello por lo que decidimos aventurarnos en la realización de esta experiencia de servicio a la comunidad cuyo punto de partida se fundamenta en el concepto de justicia social que a continuación desarrollamos más detenidamente. 
Uno de los factores clave para conseguir desarrollar sociedades más justas es contar con una educación de calidad. Para ello, es fundamental llevar a cabo un cambio equitativo de la misma, donde se desarrolle una buena y coherente formación en y para la justicia social.

No es fácil definir, y mucho menos explicar, dicho término debido al carácter polisémico que el transitar por diferentes contextos le otorga. Para ello, nos basamos en la comparación que Griffiths (2003), de una manera muy genérica, hace del mismo con un verbo. Dicho término se entiende como un proceso dinámico, nunca completo, sujeto a reflexión y mejora, de repartición justa y equitativa de los bienes y servicios fundamentales necesarios para el correcto desarrollo y adecuado desenvolvimiento de las personas en la sociedad, con el fin de procurarles una mayor calidad de vida, basada en el aumento de oportunidades y de superación personal.

Esta definición, si bien es acertada, resulta incompleta, ya que únicamente cita el concepto de Distribución (Rawls, 1971; Sen, 2010), sin tener en cuenta otras dimensiones como el Reconocimiento (Fraser, 1998; Cole, 2000; Irvine, 2003) y la Representación (Miller, 1999; Bell, 1997; Harnett, 2001; Lee e Hipólito-Delgado, 2007), siendo estas concepciones claves en la fundamentación teórica sobre la que se basa el término multidimensional de justicia social (Fraser, 2008).

De manera que, en términos generales, y basándonos en las definiciones realizadas por Fraser (2008), Fraser y Honneth (2003) y Griffith (2003), podríamos decir que la justicia social es un proceso dinámico sujeto a reflexión y mejora, orientado a garantizar la igualdad de oportunidades, mediante la reformulación de nuevas políticas económicas y sociales, que aseguren una adecuada distribución de los bienes y servicios fundamentales, confirmando el reconocimiento de todas y cada una de las identidades, y posibilitando su plena representación en la vida social.

Visto esto y teniendo muy presente la influencia del modelo neoliberal, percibimos una sociedad y una educación que no contemplan, o por lo menos no de manera relevante, el término justicia social. Esta situación, junto con la relación que guardamos con el ámbito educativo donde desarrollamos nuestra práctica profesional y lo expuesto por Freire (1978: 3) sobre la importancia de la educación como praxis, reflexión y acción del hombre sobre el mundo para transformarlo, nos ha hecho replantearnos la responsabilidad que la universidad en general y sus docentes en particular tenemos en el proceso de recuperación social.

Vemos conveniente introducir en la universidad metodologías que posibiliten romper con el modelo social vigente e incorporar prácticas que integren adecuadamente la preparación para el desarrollo de la práctica profesional, el ejercicio de la ciudadanía activa y la responsabilidad social de sus estudiantes.

Visto esto y valiéndonos de lo propuesto por Aramburuzabala (2015), creemos que integrar metodologías como las del Aprendizaje Servicio pueda ayudar en la consecución de tales propósitos porque permiten, como herramienta de aprendizaje y de transformación social al estudiantado, cultivarse mientras actúan intentando cubrir las diversas necesidades del entorno con el objetivo de transformar así la rea- 
lidad mediante acciones de mejora, al tiempo que reflexionan de forma estructurada sobre la experiencia vivida.

\section{LA METODOLOGIA DE ApS COMO MARCO DE ESTA EXPERIENCIA}

Para Martínez (2008), las universidades están comprometidas con la sociedad y es por ello por lo que están incorporando a su tarea institucional la responsabilidad social como uno de sus pilares fundamentales. Pero creemos que esto no es suficiente. Creemos que no solo debemos introducir nuevas metodologías, sino que tenemos que facilitar que se desarrollen. Necesitamos una formación que aúne conocimientos, métodos y valores mediante las vivencias facilitadas por el contexto al que se adscriben. Esto supone que dicha formación, además de contemplar contenidos teóricos y metodológicos recomendables para la práctica docente, también albergue contenidos prácticos obtenidos de la capacidad de crítica, de reflexión y de participación activa que el contacto con la realidad facilita. García y Cotrina (2015) afirman que, junto con el prácticum, la utilización del ApS durante la formación del profesorado dota al alumnado de un perfil profesionalizante de enorme valor fruto de la participación activa del estudiantado en propuestas y proyectos en concreto.

Igualmente, y parafraseando a Martínez (2008: 82), «la acción, la práctica, también es fuente de conocimiento de manera que no basta solo con trabajar la dimensión cognitiva de los alumnos ya que la manera de aprender es, en gerundio, haciendo".

Así pues, creemos que la utilización del ApS puede considerarse una herramienta de enorme relevancia durante la formación del futuro profesorado en los procesos de construcción de sociedades más justas, ya que según lo expuesto por Aramburuzabala, García-Peinado y Elvias (2013), el ApS es una innovadora práctica educativa que combina objetivos de aprendizaje curricular con objetivos de servicio a la comunidad, con la intención de mejorar las realidades donde se realiza el servicio, y que considera a quien recibe el servicio como un elemento central y no como un simple receptor de actos de caridad. Estos elementos favorecen el desarrollo del pensamiento crítico y la conciencia social que convierten las actividades de servicio en experiencias transformadoras, y a los estudiantes en agentes de cambio con una visión de un mundo más justo que trabajan activamente para crearlo.

Del mismo modo, Puig (2009) afirma que el ApS es una metodología pedagógica pluridimensional con un gran poder formativo que aglutina en una única actividad el aprendizaje de contenidos, competencias y valores, con el desarrollo de tareas de servicio a la comunidad, con el propósito de mejorar algo en la misma. El aprendizaje y el servicio de esta metodología, además de complementarse mutuamente (figura 1), quedan fuertemente vinculados por una relación circular que les aporta beneficios; porque lo teórico del aprendizaje adquiere el realismo de la vivencia y el realismo de la vivencia adquiere un marco teórico sobre el que fundamentarse en la construcción del conocimiento. 


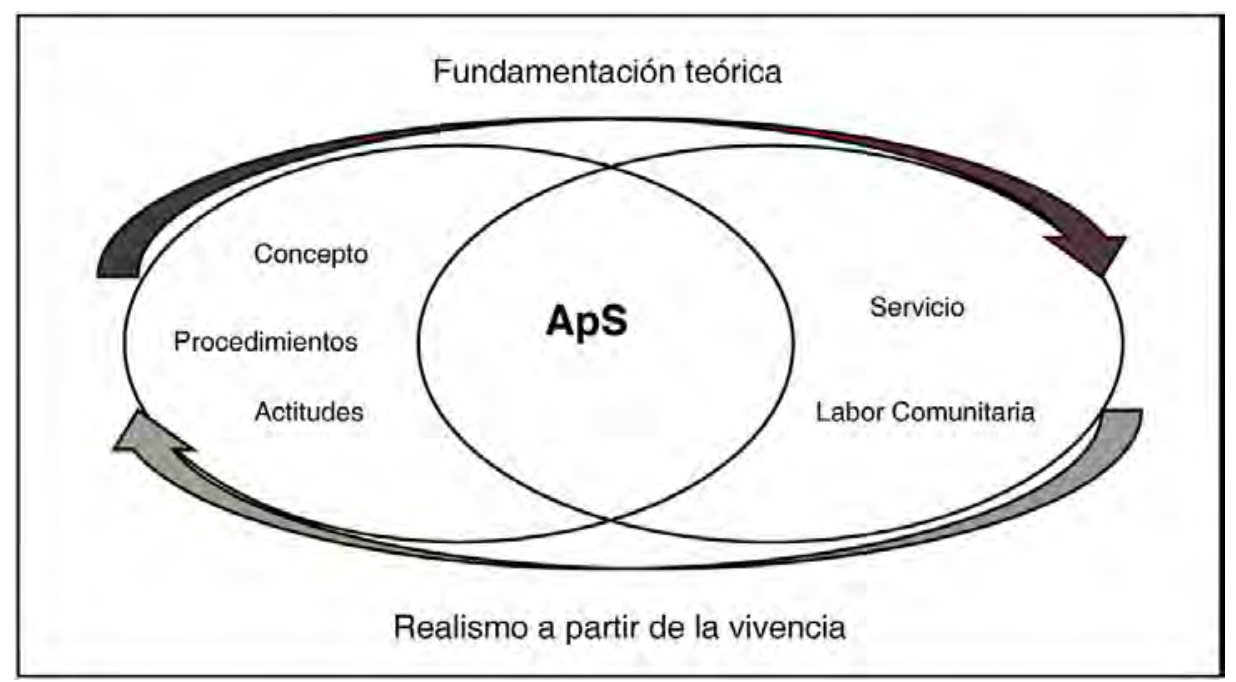

Fuente: elaboración propia.

Figura 1. Esquema del proceso de Aprendizaje Servicio (ApS).

Muchas son las definiciones, nacionales e internacionales, que hacen referencia al termino ApS, pero parece que todas ellas señalan a dos como los componentes básicos del mismo: el aprendizaje eficaz y de calidad de conceptos, procedimientos y actitudes ligados al currículum, y el servicio y su labor comunitaria que converge en una transformación comunitaria (Francisco y Moliner, 2010).

Hablamos pues de una metodología viva, real, dinámica; en definitiva, activa, que permite aprendizajes contextualizados, difíciles de encontrar en entornos fuera del ámbito en que se desarrolla la acción, pues pierden la visión global que les presta el entorno que los rodea. En definitiva, hablamos de una metodología que puede facilitar la transgresión de las posiciones hegemónicas sobre el aprender y el enseñar hasta ahora utilizadas en general en el contexto educativo y más concretamente en el contexto universitario.

\section{PARTICIPANTES Y CONTEXTO DE LA EXPERIENCIA}

Los participantes que hemos intervenido en el desarrollo de esta experiencia pertenecen a colectivos diferentes. En primer lugar, tenemos al alumnado y al profesorado de cuarto curso del Grado de Magisterio de Educación Infantil y Primaria en la mención de Educación Intercultural, miembros integrantes de la comunidad escolar y, por último, pero no por ello menos importante, asociaciones de vecinos de diferentes barrios, todos ellos residentes en la ciudad de Vitoria-Gasteiz. 


\section{DESCRIPCIÓN DE LA EXPERIENCIA REALIZADA}

La experiencia se ha desarrollado durante el curso 2016-2017, en la asignatura "gestión de proyectos específicos», impartida durante el segundo cuatrimestre del 4. ${ }^{\circ}$ curso del Grado de Magisterio Educación Infantil y Primaria en la especialidad de Educación Intercultural, con un grupo de 54 alumnos en la Facultad de Educación y Deporte de la Universidad del País Vasco, ubicada en la ciudad de Vitoria-Gasteiz, perteneciente a la provincia de Álava, dentro de la Comunidad Autónoma Vasca del Estado español.

A través de la misma, nuestro alumnado ha desarrollado las competencias tanto generales como específicas de la asignatura, realizando varios servicios en diferentes barrios-comunidad de vecinos de Vitoria-Gasteiz.

La duración de esta experiencia ha sido de 14 semanas, es decir, lo correspondiente al segundo cuatrimestre, repartida en tres fases principales (tabla 1).

\begin{tabular}{ccl} 
& TABLA 1. TEMPORALIZACIÓN DE LA EXPERIENCIA DE ApS \\
\hline $1 .{ }^{a}$ FASE & 1-3 semanas & $\begin{array}{l}\text { - Acercamiento teórico al concepto de ApS } \\
- \text { Explicación de la experiencia }\end{array}$ \\
\hline 2. ${ }^{\text {F FASE }}$ & 3-12 semanas & $\begin{array}{l}\text { - Observación del contexto e identificación de carencias-necesidades por } \\
\text { parte del alumnado } \\
\text { - Diseño del proyecto e implementación del mismo }\end{array}$ \\
\hline 3. ${ }^{a}$ FASE & 12-14 semanas & - Evaluación del proceso llevado a cabo y presentación del mismo \\
\hline
\end{tabular}

Fuente: elaboración propia.

\subsection{QuÉ es, PARA QUÉ y Cómo el ApS (FASE INICIAL)}

En esta fase realizamos una primera toma de contacto con la metodología a tratar. Por parte del profesorado hicimos una inmersión en los aspectos conceptuales del ApS, y posteriormente explicamos las cuestiones que implicaba el desarrollo de un proyecto de estas características, basándonos para ello en las diferentes tareas propuestas en la preparación de un programa de ApS (Puig, Batle, Bosch y Palos, 2007; Puig, 2008; Puig, Martin \& Batle, 2008; RMC Research Corporation, 2006).

En primer lugar, presentamos los principios básicos del programa en cuestión, como, por ejemplo: lugar donde se iba a desarrollar la actividad, la duración de la misma, el establecimiento de los horarios y la creación de grupos de trabajo y asignación de las diferentes zonas de trabajo que a continuación presentamos mediante la siguiente tabla (tabla 2).

A continuación, explicamos a cada grupo que debía realizar un análisis exhaustivo del contexto de la situación inicial, es decir, un análisis de la diversidad cultural del barrio para posteriormente hacer un estudio exploratorio con su respectivo diagnóstico sobre las relaciones existentes entre los miembros de las diferentes culturas que conformaban el barrio, tanto migrantes como no, y poder detectar 


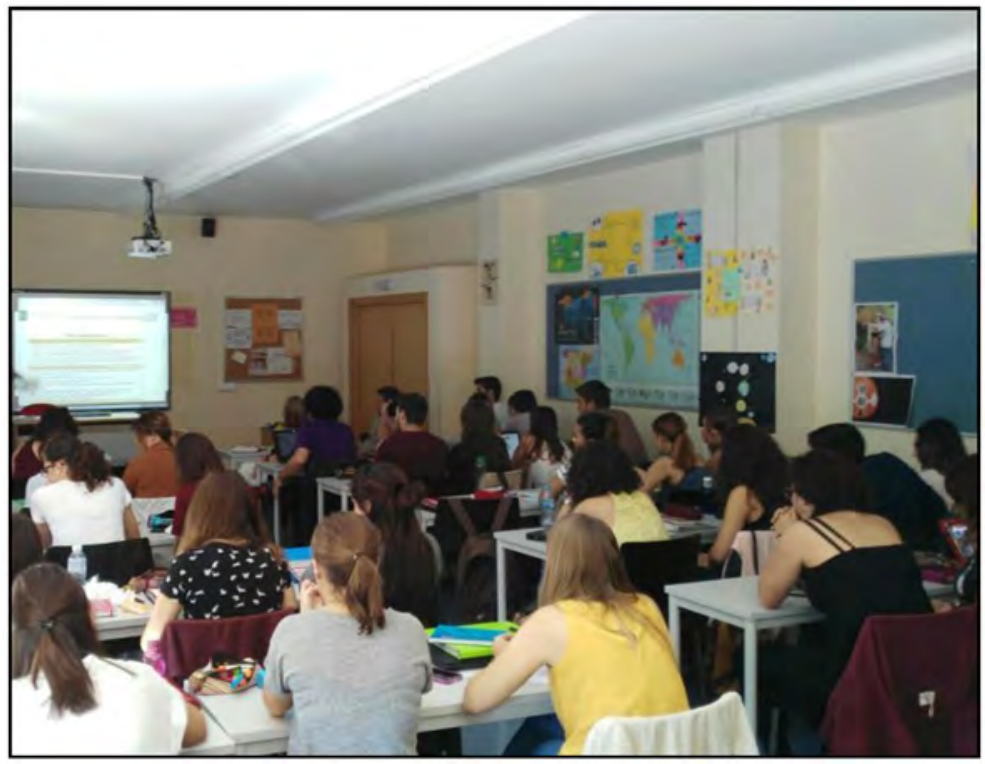

Fuente: elaboración propia.

Figura 2. Primera toma de contacto con la metodología de ApS.

así necesidades específicas de los vecinos del barrio en cuestión para, mediante la metodología de ApS, poder ofrecer un servicio que pudiera satisfacer en la medida de lo posible esas necesidades.

\begin{tabular}{|c|c|c|c|}
\hline \multicolumn{4}{|c|}{$\begin{array}{l}\text { TABLA 2. ESQUEMA RESUMEN DE LA ASIGNACIÓN DE ZONAS } \\
\text { DE TRABAJO A LOS DIFERENTES GRUPOS }\end{array}$} \\
\hline Grupo de trabajo & BARRIO ADJUDICADO & INSTITUCIÓN ESCOLAR & Asociación \\
\hline Grupo 1 & Casco Antiguo & Ramón Bajo & $\begin{array}{l}\text { - Radix } \\
\text { - Berakah } \\
\text { - Errefuxiatuak ongi etorri } \\
\text { - Adsis } \\
\text { - Zas espacio } \\
\text { - Gasteiz irekia } \\
\text { - Gasteiz txiki }\end{array}$ \\
\hline Grupo 2 & El Pilar & $\begin{array}{l}\text { Divino Maestro } \\
\text { Luis Elejalde }\end{array}$ & $\begin{array}{l}\text { - Colombia Euskadi } \\
\text { - Solidaridad internacional }\end{array}$ \\
\hline Grupo 3 & Salburua & $\begin{array}{l}\text { Salburua } \\
\text { Katagorri }\end{array}$ & $\begin{array}{l}\text { - Aintzira senideak } \\
\text { - Burdinbide }\end{array}$ \\
\hline Grupo 4 & Judizmendi & CEIP Judizmendi & - Casa de Cultura \\
\hline
\end{tabular}




\begin{tabular}{|c|c|c|c|}
\hline Grupo 5 & Coronación & $\begin{array}{l}\text { Ikastola Landazuri } \\
\text { Niño Jesús } \\
\text { Presentación María }\end{array}$ & $\begin{array}{l}\text { - Por una vida digna } \\
\text { - Casa de cultura }\end{array}$ \\
\hline Grupo 6 & Zabalgana & Colegio Zabalgana & $\begin{array}{l}\text { - Hirukide } \\
\text { - Asociación de vecinos } \\
\text { - Berribide scout } \\
\text { - AMPA Mariturri }\end{array}$ \\
\hline Grupo 7 & Zaramaga & $\begin{array}{l}\text { Paulo Freire } \\
\text { Odon de Apraiz } \\
\text { Mercedarias } \\
\text { Koldo Mitxelena } \\
\text { Escuela infantil } \\
\text { Egibide }\end{array}$ & $\begin{array}{l}\text { - Comercios y servicios } \\
\text { - Zaramagatarrak } \\
\text { - Bizigarri } \\
\text { - Nueva Vida } \\
\text { - Cáritas }\end{array}$ \\
\hline Grupo 8 & Adurtza & $\begin{array}{l}\text { Adurza ikastola } \\
\text { San Ignacio }\end{array}$ & $\begin{array}{l}\text { - Servicios sociales } \\
\text { - Insola }\end{array}$ \\
\hline Grupo 9 & Sansomendi & $\begin{array}{l}\text { Lakuabizkarra } \\
\text { Txantxangorri } \\
\text { San Prudencio } \\
\text { Paula Montal } \\
\text { IPI Sansomendi }\end{array}$ & $\begin{array}{l}\text { - Gao Lacho Drom } \\
\text { - Kalearte }\end{array}$ \\
\hline Grupo 10 & Arana & $\begin{array}{l}\text { Lopez de Guereńuq } \\
\text { Escuela Arana }\end{array}$ & $\begin{array}{l}\text { - RASD } \\
\text { - Cáritas }\end{array}$ \\
\hline
\end{tabular}

Fuente: elaboración propia.

Para finalizar con esta primera fase, presentamos los objetivos que pretendíamos conseguir con el desarrollo de esta experiencia. Por un lado, los centrados en el aprendizaje del alumnado como: (1) diseñar, planificar e implementar procesos de enseñanza-aprendizaje, en colaboración con otros profesionales, atendiendo a contextos socioculturales cambiantes para conocer la naturaleza de la sociedad en la que vivimos y poder tender así puentes entre nosotros y (2) favorecer la manifestación de una conciencia colectiva en pro de la justicia social que transcienda en su desempeño profesional configurando así su identidad como docentes. Y por otro los centrados en el servicio a la comunidad: (1) conocer la realidad cultural que se da en los barrios para identificar posibles necesidades-carencias y ofrecer un servicio que pueda satisfacerlos.

\subsection{FASE DE ACCIÓN}

Una vez finalizada esta primera toma de contacto, se llevó a cabo la fase que implicaba relacionarse directamente con las diferentes entidades, es decir, el servicio. Esta fase se caracteriza por que se divide, a su vez, en dos periodos, el de identificación de necesidades y el de desarrollo de intervenciones para cubrir dichas necesidades. 


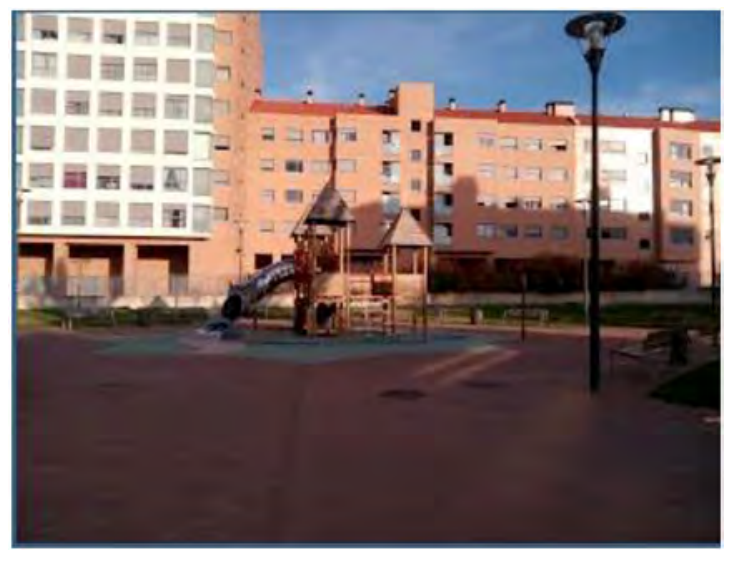

Fuente: elaboración propia.

Figura 3. Parque donde se llevó a cabo la observación.

\subsubsection{Identificación de necesidades}

Los diferentes grupos de alumnos fueron a los barrios que se les asignaron para llevar a cabo tareas de observación y de identificación de necesidades:

Con la intención de llevar a cabo un proyecto basado en el aprendizaje y servicio en un barrio de Vitoria-Gasteiz, el primer paso a seguir fue acercarnos a ese barrio para conocerlo, así como el día a día de su gente, y de este modo identificar aquellas carencias o necesidades en las que nuestro grupo de trabajo pudiera ayudar. En este primer contacto concertamos varias reuniones con diferentes colectivos del barrio: asociación de vecinos, colegios, grupos de tiempo libre, miembros del AMPA... (Grupo 1).

Una vez realizadas las labores de observación, varias fueron las necesidades identificadas que guardaban relación con las temáticas de multiculturalidad e interculturalidad recogidas mediante diferentes técnicas: observación y entrevistas, tal y como queda evidenciado a continuación:

Mediante procesos de observación:

- En el parque:

Fuimos al parque grande del barrio y nos dimos cuenta que en ese momento había 12 niños y niñas jugando divididos en dos grupos. El primer grupo era de 7 niños y nińas, todos ellos hijos de personas migrantes. No muy lejos de ellos se encontraba el otro grupo de 5 niños y niñas, todos nacidos en Vitoria. Nos resultó curioso que no estuvieran jugando juntos, al final son niños y niñas. También vimos junto con estos nińos que había tres madres, dos de las cuales eran de Vitoria y estaban sentadas juntas mientras que la otra mujer que llevaba velo y era migrante estaba sentada sola en otro banco (Grupo 1). 


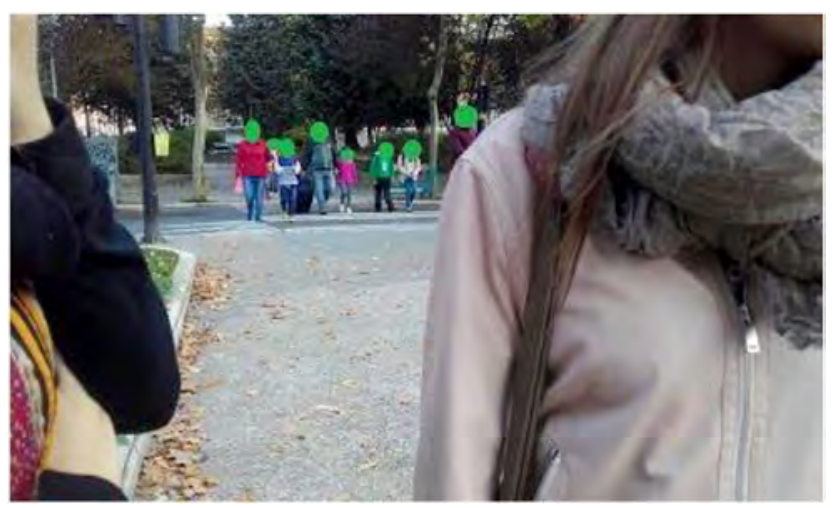

Fuente: elaboración propia.

Figura 4. Alumnado y acompańantes que se dispones a entrar en el colegio.

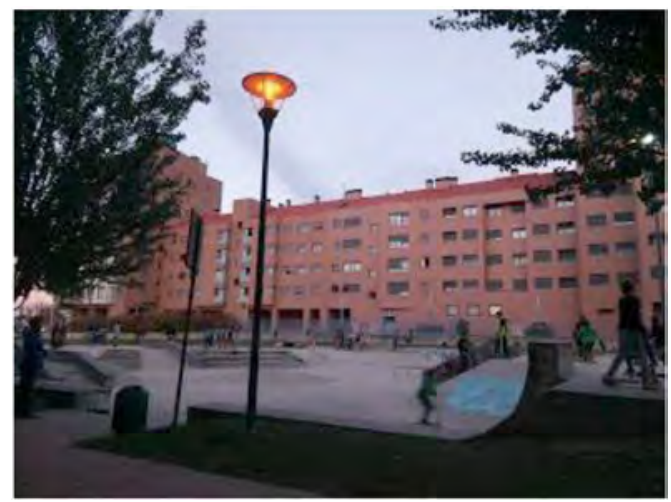

Fuente: elaboración propia.

Figura 5. «Skate park» donde se llevó a cabo la observación.

- En la puerta del colegio:

Hoy nuestro grupo ha decidido reunirse a las 8:30 de la mańana para observar el momento en el que el alumnado va al colegio y conocer así: su naturaleza, de quiénes vienen acompañados y cómo se relacionan.

Para empezar y teniendo en cuenta el origen del alumnado hemos visto que mientras que el alumnado autóctono llega acompañado de algún familiar, los alumnos de origen extranjero la mayoría llegan solos, y los pocos que van a acompañar a sus pequeños no se relacionan como lo hacen los familiares de los alumnos de Vitoria (Grupo 2).

- En el «skate park»:

En este parque vimos que había un grupo de 30 niños y niñas que estaban jugando. La mayoría de los niños de ese grupo era de aquí, pero aun y todo, había muchos niños y niñas de Marruecos y algún otro de raza negra. En este caso todos los niños estaban jugando juntos sin ningún tipo de problemas ni señales de marginación (Grupo 1). 
Mediante testimonios:

En este apartado el alumnado recoge las declaraciones de profesionales de distintas entidades institucionales: instituciones escolares y servicios sociales, sobre las características y el tratamiento que se dispensa a personas de diferentes culturas.

- Reunión con los representantes de las instituciones escolares:

En la reunión que tuvimos con un miembro de la junta directiva, ésta nos comentó que el $80 \%$ del alumnado del colegio era alumnado extranjero de 17 países diferentes y con 8 lenguas maternas diferentes por lo que la convivencia se les hacía bastante difícil.

Nos comenta que en su día había una persona responsable de los procesos de integración de las familias y del alumnado recién venido, que hacía un trabajo muy importante, pero que hace poco desde la delegación de educación decidieron prescindir de sus servicios y encomendar estas labores de integración a los propios tutores de los alumnos.

- Reunión con los servicios sociales:

La responsable de los servicios sociales nos ha comentado que en Vitoria en todos los centros cívicos existe un grupo de servicios sociales. Referente a la creación de los ghettos en Vitoria, ella nos comenta que somos nosotros los que fomentamos su creación, porque los ciudadanos de Vitoria nos sentimos muy elitistas y todos los problemas que han surgido como consecuencia de la crisis, como la disminución del trabajo y el aumento de la delincuencia, lo atribuyen al aumento de población extranjera.

Por otro lado, nos comenta que la disminución de la calidad de los servicios sociales se debe en gran medida al cambio de gobierno (derecha).

A través de entrevistas a diferentes vecinos. En este apartado el alumnado agrupa la información recogida tras realizar diversas preguntas sobre la existencia de diversidad cultural, el nivel relacional entre personas de diferentes culturas, el grado de integración en el barrio y posibles propuestas para, en caso de ser necesario, mejorar este proceso.

- Entrevistas a diferentes vecinos:

En este apartado el alumnado recoge los testimonios de diferentes vecinos del barrio sobre la diversidad cultural y las relaciones entre las diferentes culturas existentes en el mismo.

No hay interculturalidad (relación) pero, sin embargo, hay diversidad cultural porque hay mucha gente de diferentes culturas, pero en ghettos.

Yo en cambio tengo un amigo que es chino. En el barrio suelo comprar en las tiendas tradicionales y nunca he comprado en tiendas de extranjeros. Creo que la predisposición de la gente va en función de la edad porque los jóvenes no tenemos ningún problema para relacionarnos con ellos y en cambio los adultos no están dispuestos a ello (Vecino 1 Vitoria-19 años). 
No hay interculturalidad. Hay muchas personas de origen extranjero como, por ejemplo: árabes, africanos, chinos, pero no se mezclan entre ellos. Yo en cambio me relaciono con todo el mundo sin tener en cuenta su país de origen, aunque no tengo ningún amigo extranjero. Además, yo también soy inmigrante, porque vine de Burgos a Vitoria en busca de trabajo. Con respecto a las compras, las hacemos en las tiendas tradicionales. En lo que respecta a la predisposición de la gente otras personas pueden tener problemas para relacionarse con ellos, pero yo no. Para terminar, no creo que el barrio necesite ningún tipo de necesidad especial, es un barrio muy bonito y todos nos conocemos (Vecino 2 Burgos-74 ańos).

En el barrio nos juntamos personas de diferentes culturas: árabes, italianos, franceses, españoles, vascos, asiáticos y africanos y sí que tenemos relación los unos con los otros. He visto todo tipo de relaciones entre las personas de diferentes culturas, pero creo que se pueden mejorar. En el barrio he visto muchas tiendas dirigidas por personas de otros países (bares de chinos, carnicerías árabes y kebab). Yo compro en cualquier sitio, pero muchas veces compro donde los chinos porque son más baratos. También he oído algún que otro comentario racista de la gente autóctona pero normalmente suelen ser malos entendidos, suelen ser episodios sueltos. También he oído comentarios así entre personas extranjeras. Yo tengo una tienda y por eso mantengo relación con cualquiera porque a mi tienda vienen personas de todas las culturas. Para terminar, propongo que se haga algún tipo de fiesta en la que las personas de diferentes culturas cocinen algún plato de su país, se vistan como se visten en su país, traigan objetos típicos de su país para conocernos mejor entre todos (Vecino 4 Senegal-52 ańos).

He visto mucha gente de diferentes países y creo que hay mucho respeto, pero esto nos lleva a que haya diversidad cultural y no interculturalidad. Veo a los pakistaníes y a los marroquíes muy arraigados a sus culturas, pero también me parece muy respetable. Yo voy a reuniones donde nos juntamos personas de diferentes países y por tanto he creado muchos vínculos en el barrio. También veo que hay muchas tiendas extranjeras en el barrio y muchos locutorios. Yo compro en cualquier tienda en función del tiempo y del dinero del que disponga. Sí que he visto que algunos gitanos y marroquís rompen cosas y hacen pintadas en los edificios. Como propuesta para el barrio creo que hay que cambiar la educación que se da en las escuelas, en la calle y en las casas para entender todas las culturas y tener una actitud más abierta (Vecino 5 Argentina-64 años).

En el barrio se dan muchas situaciones de racismo porque los de aquí tratan muy mal a los extranjeros. Esta falta de interculturalidad es por la crisis y yo como marroquí que soy veo que a los de aquí no les importa nuestra situación. Yo las compras las hago en cualquier tienda, pero sobre todo porque son más baratas y por mi religión porque compro la carne en carnicerías árabes, pero aun y todo no tengo problemas para ir a otras tiendas a comprar. En mi tienda alguna vez he oído comentarios feos sobre personas de otras razas y eso no me gusta. No tengo muchas relaciones, las que mi trabajo en el bar me da (Vecino 9-Marruecos-55 años). 


\subsubsection{Propuestas de intervenciones para las necesidades}

En este apartado recogemos la implementación del propio servicio por parte del alumnado. Esta propuesta de intervención está fundamentada en unos objetivos formulados en base a unas necesidades identificadas mediante la observación y las diferentes entrevistas.

La principal carencia identificada por el alumnado fue la falta de relaciones humanas entre personas de diferentes culturas, es decir, la falta de interculturalidad. Los alumnos reconocían la riqueza cultural del barrio, afirmaban que se habían dado procesos de integración, pero remarcaban que no eran suficiente:

Nos gustaría conseguir un barrio sin prejuicios ni estereotipos, llegando a ser un barrio intercultural y no simplemente multicultural (Grupo 1).

Del mismo modo, comentan que les gustaría ir más allá y realizar labores de concienciación social, que la ciudadanía en general supiera y las personas del barrio en particular aceptaran lo desconocido y evitar así comportamientos, expresiones y prácticas propias de la xenofobia:

Nos gustaría destruir el miedo a lo desconocido, para poder acabar con el racismo (Grupo 1).

Visto esto, su propuesta para acabar o por lo menos disminuir las necesidades identificadas fue realizar un mural intercultural

\subsubsection{Desarrollo de la propuesta de intervención}

Teniendo en cuenta la incertidumbre en la que vivimos, que asigna una fecha de caducidad a todo, nuestros alumnos decidieron realizar algo atractivo y vistoso que se mantuviera en el tiempo:

El proceso para lograr el mural intercultrual en el barrio de Adurtza fue largo pero efectivo. Lo primero fue pensar y plantear una intervención. Para ello decidimos que queríamos realizar algo duradero, colorido y reivindicativo. Todas estas cualidades las cumplía el realizar un mural, y, por lo tanto, decidimos llevarlo adelante (Grupo 1).

Querían representar la realidad de la sociedad y del barrio en el que desarrollaron la actividad mediante un dibujo. En el mismo y como si fueran las dos caras de una misma moneda, querían explicitar por un lado la no relación entre las personas de diferentes culturas y por otro pretendían expresar los aspectos positivos de la diversidad en la sociedad contemporánea, porque, tal y como afirma Touraine (1997), la igualdad y la diferencia no solo no son contradictorias, sino que además son inseparables la una de la otra, porque una sociedad sin diferencias reconocidas sería una dictadura. 

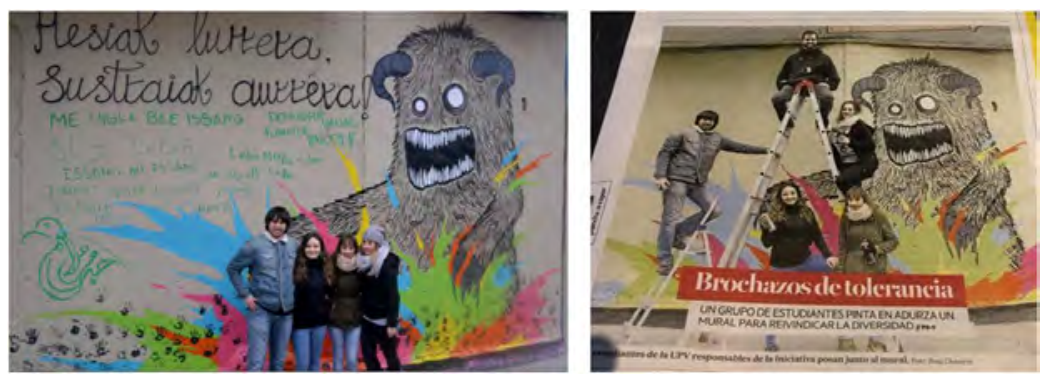

Fuente: elaboración propia.

Figuras 6a y b. Resultado del desarrollo de la actividad y su publicación en los medios de comunicación.

Lo primero fue realizar un boceto. En el boceto quisimos poner un monstruo, el cual representaría el racismo. Atravesando éste muchos rayos de colores, los cuales representarían la diversidad cultural del barrio. Además, quisimos escribir en varias lenguas madre del barrio la siguiente frase, la cual es tanto reivindicativa como muy significativa: «Hesiak aurrera, sustraiak aurrera», la cual en castellano es traducida en «derribando vallas, floreciendo raíces» (Grupo 1).

El estudiantado se puso en contacto con diferentes representantes: comunidad educativa, servicios sociales, comunidad de vecinos, etc., para fijar la fecha en la que se desarrolló la actividad y asegurar así una acción comunitaria como camino para la integración social y la interculturalidad (Vecina, 2013).

Después de un mes de búsqueda activa, logramos el permiso de una persona que nos dejaba la pared exterior de su lonja. Ahora nos tocaba fijar fecha para realizarlo e invitar a las escuelas y asociación que queríamos involucrar. Para ello, nos pusimos en contacto con la directora y el director de ambos centros del barrio (Adurtza y San Ignacio) y con la responsable de la asociación Insola. La fecha estaba fijada, los horarios para que acudiesen a colaborar, también (Grupo 1).

Por fin llego el día en el que el estudiantado y diferentes agentes de la comunidad se pusieron «manos a la obra» y realizaron el mural. Querían realizar la actividad y difundirla a través de diferentes medios de comunicación para generar resonancias en los receptores, porque, según Castells (2008), los medios de comunicación son decisivos en la formación de la opinión pública que condiciona la decisión política.

Y por fin llegó la fecha. El 31 de enero nos levantamos bien temprano para empezar con el mural. Durante la mañana estuvimos pintando el mural, pero también hablando con la radio y prensa. Que acudieran estas dos últimas no fue casualidad, ya que días antes de realizar el mismo, acudimos a sedes de ambos medios para hablarles sobre el proyecto. Nuestro objetivo era que la noticia se hiciese eco en toda la ciudad, y quién sabe, podía ser que en algún otro barrio de Vitoria alguien aportase su granito de arena para que la ciudad entera se llene de gente que trabaja a favor de la interculturalidad e igualdad entre personas (Grupo 1).

Mostramos el resultado de todo este proceso mediante las figuras 6 a y b. 


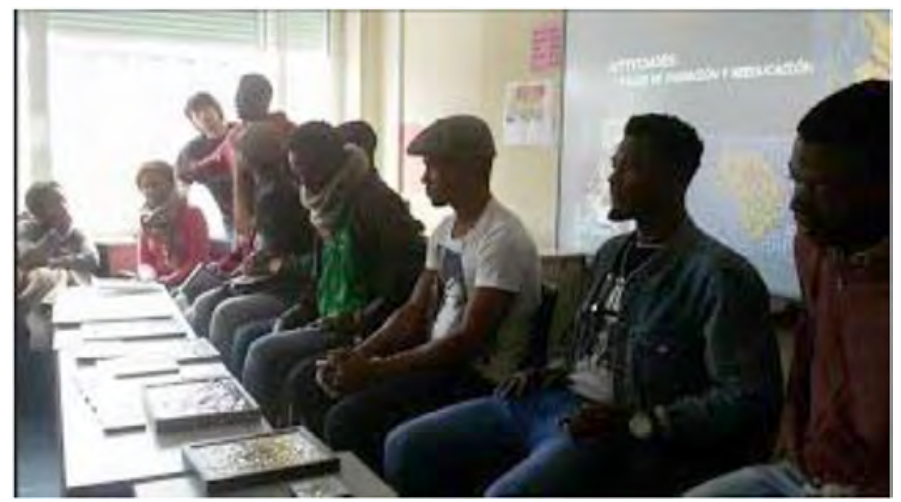

Fuente: elaboración propia.

Figura 7. Miembros de la comunidad donde se desarrolló la actividad realizando el feedbak al alumnado en la universidad.

\subsection{FASE FINAL}

En esta fase de valoración, se llevaron a cabo diferentes subfases: la demostración de los conocimientos adquiridos por parte del alumnado mediante la presentación de lo implementado y la evaluación del mismo.

La evaluación del apartado del «aprendizaje» de la experiencia por parte del alumnado se realizó en la universidad mediante una presentación del ApS, diseñado, implementado y evaluado frente a un tribunal compuesto por tres profesores de la asignatura donde se desarrolló esta experiencia. El propósito de esta actividad era la de esclarecer la adquisición de conocimientos por parte del alumnado.

Igualmente, la evaluación del apartado «servicio» la realizaron algunos miembros de la comunidad donde se desarrolló la actividad, junto con los alumnos participantes, para poder conocer así el nivel de satisfacción con respecto a los objetivos previamente planteados. Fue un proceso muy interesante de aportaciones y devoluciones que presentamos a continuación mediante diferentes testimonios y la figura 7.

Ha sido una experiencia muy bonita que ha servido para poder conocer a más gente y para que más gente nos pudiera conocer (Participante en la actividad y miembro de la comunidad africana).

Esta experiencia nos ha servido para que la gente de aquí deje de tratarnos como niños porque nosotros también tenemos cosas que aportar (Participante en la actividad y miembro de la comunidad africana).

\section{RESULTADOS}

En este apartado hemos intentado recoger testimonios de diferentes alumnos y alumnas sobre la repercusión que la utilización de este tipo de metodologías tiene en sus procesos de enseñanza-aprendizaje. De igual manera y teniendo muy presente 
la vinculación que para nosotros tiene la implementación de este tipo de metodologías con el desarrollo de sociedades más justas-justicia social, hemos querido plasmar la visión que el alumnado tiene al respecto, mediante la compilación de diferentes testimonios que intentan dar respuesta a dos cuestiones planteadas por el equipo docente.

\subsection{El ApS como facilitador del proceso enseñanZa-Aprendizaje}

A continuación, mostramos varias razones por las que nuestros educandos apuestan por la utilización de la metodología de $\mathrm{ApS}$ como herramienta facilitadora en y para sus procesos de enseñanza-aprendizaje.

Hemos podido conocer la gente que habita este barrio, la gente que trabaja en él y la gente que por más que lo intente, no consigue una visibilidad y una interacción real en el barrio (personas migrantes). Una vez detectadas las necesidades, estuvimos más que preparados para proponer y llevar a cabo una intervención $\left(\mathrm{A} 1 \_\mathrm{F}^{1}\right)$.

Entre las razones por las que han decidido denominar a la metodología de ApS como herramienta facilitadora, predomina la posibilidad que esta ofrece para conocer la realidad del contexto en cuestión y poder desarrollar así actividades más acordes con su casuística.

Lo que me parece más interesante de esta metodología es que la teoría y la práctica se fusionan y se necesitan entre sí. No se entiende una intervención sin la exploración del barrio, ni una exploración en vano. Ya que ambas van de la mano, nos han servido para trabajar mano a mano con la gente del barrio, y además, para involucrar e informar sobre esta realidad a mucha gente que conforma el barrio. En la universidad debería haber más de esto y no tanta teoría que si no la experimentamos no sirve de nada (A2_M²).

Comentan el carácter práctico que tiene realizar este tipo de actividades, frente a las realizadas en la universidad, fundamentadas únicamente en aspectos teóricos. Inciden en la mayor capacidad para adquirir y transmitir el continente y el contenido del mensaje estando inmersos en el propio contexto. Hablan de la importancia de los procesos activos en la adquisición de conocimientos, pues estos van a condicionar sus acciones futuras (Lemke, 2006).

Además de un proceso de «acción-reacción», tenemos una relación y cadena humana, de la cual, lo más importante es la incondicionalidad. Ayudan y aportan sin condición, sin esperar nada a cambio, de una forma desinteresada e involucrada.

1 A1_F. Alumna número uno. Código utilizado para enumerar a los alumnos y alumnas y diferenciarlos en función del género (F: femenino y M: masculino).

2 A2_M Alumno número dos. Código utilizado para enumerar a los alumnos y alumnas y diferenciarlos en función del género (F: femenino y M: masculino). 
Este tipo de procesos o metodología, bajo mi punto de vista, son capaces de sacar lo más bello y humano de las personas (A3_F3).

De igual manera inciden significativamente en la calidad de las relaciones humanas que afloran durante el desarrollo de este tipo de actividades debido al nivel de satisfacción que experimentan en el trabajo que están desarrollando (Benito, 2006).

\subsection{Relación Entre ApS y justicia social}

En este apartado plasmamos las relaciones que según nuestro alumnado guarda la metodología del ApS con la construcción de sociedades más justas. Para ello tomamos el testimonio del alumnado de vuelta ya en la universidad, realizado de forma conjunta, como cierre de la experiencia.

Sin duda alguna, este tipo de actividades o, mejor dicho, procesos, ayudan a la hora de construir sociedades más justas. Hay muchas razones por las que creemos en ello, y algunas de ellas son las siguientes: (1) conciencian sobre las diferencias sociales existentes entre las personas por su raza, sexo, religión, color, (2) dan pie a explicar/investigar los derechos humanos de todas las personas sobre la faz de la tierra, (3) educan en un sentimiento de empatía y solidaridad, (4) demuestran que hay gente dispuesta a luchar contra estas desigualdades sociales, (5) ayudan a romper estereotipos y prejuicios, (6) dan la oportunidad para trabajar de primera mano con personas migrantes, conociendo lo desconocido, terminando con el miedo y acabando con el racismo (Alumnado de Grado de Magisterio).

Mediante esta afirmación, el alumnado remarca la importancia de esta metodología en el desarrollo de perspectivas (razones) que favorezcan el incremento de la reflexión para la acción que suponga el cambio social sobre el que se fundamenta la construcción de sociedades más justas, porque

desarrollan debates críticos sobre temas relacionados con el poder, los privilegios y las desigualdades sociales y examinan de forma crítica cuestiones como el racismo y la igualdad de oportunidades, favoreciendo el desarrollo y compromiso social de los participantes desde una perspectiva transformadora. Los estudiantes reflexionan de forma estructurada sobre estas realidades, su origen, cómo prevenirlas y afrontarlas, y sobre el impacto del servicio en la mejora de la situación de injusticia y en el cambio social (Aramburuzabala, 2015, p. 7)

Del mismo modo, el estudiantado habla de la necesidad de conocer a las personas que junto a nosotros conforman la sociedad para poder llevar a cabo procesos de (re)construcción social de manera conjunta. Para conocernos primero

${ }^{3}$ A3_F Alumna número tres. Código utilizado para enumerar a los alumnos y alumnas y diferenciarlos en función del género (F: femenino y M: masculino). 
debemos aceptarnos mutuamente y esto en palabras de Maturana (2008:27) implica «la aceptación del otro como un legítimo otro en la convivencia».

En nuestra opinión, para poder formar entre todos una sociedad más justa lo más necesario es conocerse. No se puede hablar de sociedad sin hablar de vecinos, y de la diversidad que, tal y como antes se ha explicado, tan ligada va con ellos. Una sociedad no puede llegar a ser justa si no se es consciente de las características de esta, así como de sus necesidades. Es por esto que es fundamental fijarnos en nuestro entorno, prestar algo más de atención a todo aquello que tenemos alrededor, y así poder dar respuesta a lo que la sociedad pide, para que sea lo más justa posible. Al fin y al cabo, la diversidad es enriquecimiento, y es algo de lo que debemos valernos (Alumnado de grado de magisterio).

Igualmente, remarcan el valor que la sociedad debe otorgar a la diversidad como factor significativo de incalculable valor para adquirir un aprendizaje significativo y fiscalizador (Gómez, 2002) de enorme importancia en la construcción de una sociedad democrática y acogedora de la pluralidad (Echeita, 1999; Porras, 1998; López-Melero, 1995).

\section{CONCLUSIONES}

Los procesos de cambio e innovación por lo general son beneficiosos. Teniendo en cuenta las declaraciones aportadas por el alumnado, la universidad, como institución comprometida con el cambio social, necesita realizar modificaciones en los planteamientos y en las formas de enseñar. Nuestro alumnado demanda experimentar un modelo de formación verdaderamente comprometida con la sociedad y con ellos mismos. Necesitan una formación que les facilite poder desarrollarse como personas justas, críticas, comprometidas con la comunidad, indistintamente del contexto de su localización, es decir, una educación desde y para la justicia social. Solicitan un modelo de formación innovadora cuyo objetivo fundamental sea la adquisición del aprendizaje significativo del alumnado nutriendo, para ello, ámbitos tan importantes como el personal, el social y el profesional teniendo en cuenta el poder de la comunidad en los procesos de enseñanza-aprendizaje.

Igualmente, la apertura e inmersión en contextos reales, así como la colaboración con agentes activos fundamentales para el desarrollo del cambio social, ha supuesto por un lado el desarrollo competencial del alumnado mediante el realismo a partir de la vivencia, y por otro la mejora de la realidad social a partir de la fundamentación teórica.

Por ello vemos preciso considerar la fortaleza de la metodología del ApS como herramienta que responde a las necesidades de la sociedad contemporánea y proponemos utilizar este tipo de metodologías en el ámbito universitario, que discute las expectativas iniciales del pensar y del hacer abriendo nuevas sendas que cuestionan las posiciones hegemónicas del enseñar y el aprender. 


\section{BIBLIOGRAFÍA}

Alonso-Sáez, I., Arandia, M., Martínez, I., Martínez, B. y Gezuraga, M. (2015). «El aprendizaje-Servicio en la innovación universitaria. Una experiencia realizada en la formación de educadoras y educadores sociales». Revista Internacional de Educación para la Justicia Social, 2 (2), 195-216.

Alonso-SÁez, I. y Arandia, M. (2014). «Aprender creando: "Factoría Creativa” en las aulas universitarias. REDU: Revista de Docencia Universitaria, 12 (1), 443-468.

Aramburuzabala, P. (2015). Aprendizaje-servicio: Una herramienta para educar desde y para la justicia social. Revista Internacional de Educación para la justicia social, 2 (2), 5-11.

Aramburuzabala, P., García-Peinado, R. y Elvias, S. (2013). «Educación desde y para la Justicia Social: una Experiencia de Aprendizaje-Servicio en la Formación de Maestros». En M.C. Pérez y M.M. Molero (coords.), Variables psicológicas y educativas para la intervención en el ámbito escolar (pp. 257-264). Almería: Asociación Universitaria de Educación y Psicología.

Aramburuzabala, P. y García, R. (2012). «El aprendizaje-servicio en la formación de maestros». Revista del Congrés Internacional de Docència Universitària i Innovació (CIDUI), 1 (1).

Baldwin, S.C., Buchanan, A.M. y Rudisill, M.E. (2007). «What teacher candidates learned about diversity, social justice, and themselves from service-learning experiences». Journal of Teacher Education, 58 (4), 315-327.

Bauman, Z. (2007). Los retos de la educación en la modernidad líquida. Barcelona: Gedisa.

BeLL, L.A. (1997). Teaching for diversity and social justice: a sourcebook. Nueva York: Routledge.

Benito, B. (2006). "Las relaciones interpersonales de los profesores en los centros educativos como fuente de satisfacción». En Convergencia con Europa y cambio en la universidad: XI Conferencia de Sociología de la Educación.

Caride, J.A. (2008). «El Grado de Educación Social en la construcción del Espacio Europeo de la Educación Superior». Educación XX1, 11, 103-131.

Castells, M. (2008). "Comunicación, poder y contrapoder en la sociedad red (II). Los nuevos espacios de la comunicación». Telos, 75, 11-23.

Coelho, E. (2006). Enseñar y aprender en escuelas multiculturales: una aproximación integrada. Barcelona: Horsori Editorial, Sl.

Cole, M. (2000). Education, equality and human rights: Issues of gender, 'race,' sexuality, special needs and social class. Londres: Routledge/Falmer Press.

Correa, J.M. y Santamaría-Goicuria, I. (2016). «La escuela en la construcción de sociedades más justas: análisis de las prácticas ante perfiles de alumnado migrante». En Rivera-Vargas, P., Sánchez, E., Morales-Olivares, R., Sáez-Rosenkranz, I., Yévenes, C. y Butendieck, S. (coords.), Conocimiento para la equidad social: pensando Chile globalmente (pp. 421-432). Santiago de Chile

De Ferari, J.M. (2006). «Responsabilidad social universitaria aproximación al concepto y a su práctica». En J.M. De Ferari, C. Fernández y C. Delpino (coords.), Una manera de ser Universidad. Teoria y práctica de la experiencia chilena (pp. 41-43). Santiago de Chile: Proyecto Universidad: Construye País.

Delors, J. (1996). La Educación encierra un Tesoro: Informe a la UNESCO de la Comisión Internacional sobre la Educación para el Siglo XXI. Compendio: Santillana. 1996. 
Echeita, G. (1999). «Reflexiones sobre Atención a la Diversidad». Acción Educativa, (102), 103.

Francisco, A. y Moliner, L. (2010). «El Aprendizaje Servicio en la Universidad: una estrategia en la formación de ciudadanía crítica». Revista electrónica interuniversitaria de formación del profesorado, 13(4).

Fraser, N. (1998). Social justice in the age of identity politics: redistribution, recognition, participation. Discussion paper. No. FS I 98-108. Recuperado a partir de http://www.econstor.eu/ handle/10419/44061.

Fraser, N. (2008). Escalas de justicia. Barcelona: Herder Editorial.

Fraser, N. y Honneth, A. (2003). Redistribution or recognition? A Political-philosophical Exchange. Londres: Verso Press.

Freire, P. (1978). La educación como práctica de la libertad. Uruguay: Siglo XXI.

García, M. y Cotrina, M.J. (2015). «El aprendizaje y servicio en la formación inicial del profesorado: de las prácticas educativas críticas a la institucionalización curricular». Profesorado. Revista de Currículum y Formación de Profesorado, 19 (1), 8-25.

Gehring, H. (2008). «Análisis de las competencias genéricas del modelo aprendizaje basado en competencias en el diseño de las nuevas titulaciones del EEES como espacio transversal para la integración de elementos de la educación para el desarrollo», en Actas IV Congreso Universidad y Cooperación al Desarrollo, Barcelona.

Gómez, A. (2002). «Un aprendizaje diverso y relevante». Cuadernos de Pedagogía, 311, 66-70.

Griffiths, M. (2003). Action for social justice in education. McGraw-Hill Education (UK).

Hartnett, D. (2001). The history of justice. Paper presented at the Social Justice Forum, Loyola University, Chicago.

Hernández, N. (2010). «Aprendizaje Servicio: un eje articulador en la Educación en Solidaridad con las y los jóvenes desde una mirada de Responsabilidad Social». Revista Científica TZHOECOEN, 5, 126-134.

IRVINE, J.J. (2003). Educating teachers for diversity: Seeing with a cultural eye. Nueva York: Teachers College Press.

Lee, C.C. e Hipólito-Delgado, C. (2007). Counselors as agents of social justice. Counseling for Social Justice, 13-28. Alexandria: American Counseling Association.

LEMKe, J.L. (2006). «Investigar para el futuro de la educación científica: nuevas formas de aprender, nuevas formas de vivir». Enseñanza de las ciencias: revista de investigación y experiencias didácticas, 24 (1), 5-12.

López Melero, M. (1995): «Diversidad y Cultura: Una escuela sin exclusiones». Rev. Kikirikí. (38). 26-38. MCEP. Sevilla.

LuCAS, S. y Martínez-Odría, A. (2012): «La implantación y difusión del Aprendizaje-Servicio en el contexto educativo español. Retos de futuro de una metodología de enseñanza-aprendizaje para promover la innovación en la Educación Superior». Comunicación presentada en el VII CIDUI: La universidad: una institución de la sociedad, celebrado en Barcelona los días 4-6 de julio.

Martínez, B., Martínez, I., Alonso, I. y Gezuraga, M. (2013). «El aprendizaje-servicio, una oportunidad para avanzar en la innovación educativa dentro de la Universidad del País Vasco». Tendencias pedagógicas, (21), pp. 99-118. 
Martínez, M. (ed.) (2008). Aprendizaje servicio y responsabilidad social de las universidades. Octaedro-ICE.

Maturana, H. (2008). Emociones y Lenguaje en Educación y Politica. JC Saez editor. Comunicaciones Noreste.

Miller, D. (1999). Principles of social justice. Cambridge, MA: Harvard University Press. 1999.

Porras, R. (1998). «Una escuela para la integración educativa. Una alternativa al modelo tradicional». Sevilla. Cuadernos de Cooperación Educativa/MCEP.

Puig, J.M., Batlle, R., Bosch, C. y Palos, J. (2007). Aprendizaje servicio. Educarpara la ciudadanía. Barcelona: Ministerio de Educación y Ciencia, Octaedro.

Puig, J.M., Martín, X. y Batlle, R. (2008). «Com començar una experiència daprenentatge servei». Barcelona: Centre promotor d'APS en Catalunya. Recuperado de http://www.aprenentatgeservei.org/intra/aps/documents/Guia_com_comencar_REVISADA.pdf.

Puig, J.M. (2008). «Com fer APS en les entitats socials?». (en línea). Recuperado el 15/01/2010 de: http://www.aprenentatgeservei.cat/intra/ aps/documents/aps_entitats_socials3.pdf.

Puig, J.M. et al. (2009). Aprendizaje Servicio. Educación y compromiso civico. Barcelona: Graó.

Rawls, J.A. (1971). A theory of justice. Cambridge, MA: Harvard University Press.

Rizvi, F. (2010). «La educación a lo largo de la vida: más allá del imaginario neo-liberal». Revista Española de Educación Comparada, 16, 185-210.

RMC Research Corporation (2006). K-12 Service-Learning Project Planning Toolkit. Scotts Valley, CA: National Service-Learning Clearinghouse. (en línea). Recuperado el 3/02/2010 de: http://www.servicelearning.org/filemanager/download/ K-12_Service-Learning Project_Planning_Toolkit.pdf.

SAncho, J.M. y Correa, J.M. (2013). «Aprender a ser maestra: perplejidades y paradojas». Cuadernos de Pedagogía, 436, pp. 18-21.

Sen, A. (2010). La idea de la Justicia. Madrid: Taurus.

Touraine, A. y Pons, H. (1997). ¿Podremos vivir juntos?: iguales y diferentes. Madrid: PPC.

Vecina, C. (2013). «La acción comunitaria, un camino para la integración social y la interculturalidad». Trabajo Social Global. Revista de Investigaciones en Intervención Social, 3 (5), pp. 54-75. 